\title{
Prize Allocation in Contests with Size Effect through Prizes
}

\author{
Sanghack Lee ${ }^{1}$, Shi Young Lee ${ }^{2}$ \\ ${ }^{1}$ Department of International Commerce, Kookmin University, Seoul, Korea \\ ${ }^{2}$ Division of Business, Chung-Ang University, Ansung, Korea \\ Email: slee@kookmin.ac.kr
}

Received February 6, 2012; revised March 2, 2012; accepted March 10, 2012

\begin{abstract}
We examine prize allocation in contests in which the number of contenders affects prizes. The government allocates a fixed prize between two groups of contenders. The prize share of each group is determined endogenously through the inter-group share contest. Then, the contenders in each group respectively compete for the prize. Examining prize allocation in such contests, we obtain the following results. The contenders expend more resources when they cooperate than when they contribute non-cooperatively in the inter-group share contest. The size effect through prizes increases the prize share of the larger group. The larger group can even obtain the larger share if the size effect through prizes is sufficiently strong.
\end{abstract}

Keywords: Contest; Prize Allocation; Endogenous Prize Share; Size Effect

\section{Introduction}

Contests are a resource-allocating mechanism in which winners are selected by certain rules based on resources expended by contenders. ${ }^{1}$ A large set of economic, political and social interactions can be modeled as a contest. Prizes for winners vary from pecuniary prizes to nonpecuniary prizes such as pride, fame, honor, etc. Many contests are stratified in such a way that prizes are allocated among competing groups by authorities and then intra-group contests for the prizes follow. The prime example is government budget allocation among local governments, research fund allocations, and so on.

The number of contenders often affects prizes in contests. ${ }^{2}$ For example, pride and honor of winners in sporting contests would naturally increase in the number of contenders. Examining contests in which prizes increase in the number of contenders, Eggert and Kolmar [4] show that an individual utility can decrease initially and then increase with the number of contenders in such contests.

The purpose of this note is to examine prize allocation in stratified contests in which prize share of each group is endogenously determined and the number of contenders also affects prizes. The government allocates a fixed prize between two groups of contenders in proportion to the group expenditure in the inter-group contest, as in

\footnotetext{
${ }^{1}$ For a collection of contributions to the theory of rent-seeking contest, see Congleton, Hillman and Konrad [1]. Konrad [2] also offers a succinct and unified treatment of the theory of contest.

${ }^{2}$ The number of contenders can also affect costs of effort via external economies of scale. Lee [3] examined this issue.
}

Katz and Tokatlidu [5]. Then, contenders in each group respectively compete for the prize. By examining prize allocation in contests with size effects, we aim to extend our understanding of the stratified contests and design of such contests.

The main results of this note are as follows. The groups expend more in the first-stage share contest in the cooperative regime than in the non-cooperative Nash regime. This is because the larger group can mitigate the free-rider problem in the cooperative regime. The size effects through prizes mitigate the group size disadvantage of the larger group. Moreover, if the size effects are sufficiently strong in the cooperative regime, the larger group can even obtain the larger share. We also draw some policy implications regarding psychological benefits (incentives) and frames (environments) based on the afore-mentioned results.

The remainder of the note is organized as follows. Section 2 examines prize allocation in contests with size effect through prizes and draws policy implications. The final section offers concluding remarks with possible extensions.

\section{Contests with Size Effect through Prizes}

Consider an allocation by government of a fixed prize $Z$ between two groups of contenders. The groups of contenders may be regions or academic fields. The contenders may be firms, research institutions, local governments, or individuals. Total population of $N$ contenders are divided into two groups denoted 1 and 2, respectively, 
where $N \geq 3$. Group $i$ consists of $n_{i}$ contenders, where 1 $\leq n_{i} \leq(N-1)$ for $i=1,2$, and $n_{1}+n_{2}=N$. Without loss of generality, we assume that $n_{1} \geq n_{2}$.

The prize increases in the number of contenders. The more contenders participate in the contest, the larger pecuniary prize is given to the winner of the contest. Even when the pecuniary prize is fixed, social psychological prize such as honor and pride may accrue to the winner of the contest.

As in Katz and Tokatlidu [5], the contest for the prize takes place in two stages. In the first stage, the two groups engage in the inter-group contest for the prize share. ${ }^{3}$ The contenders in each group determine their contribution to the share contest either cooperatively or non-cooperatively. The shares are assumed to be proportional to the group contributions. Let $s_{i}$ denote the prize share of group $i$, where $0 \leq s_{i} \leq 1$ for $i=1,2$, and $s_{1}+s_{2}$ $=1$. Following Eggert and Kolmar [4], the prize is assumed to be augmented by group size effect. Thus, $n_{i}$ contenders of group $i$ compete for the prize $s_{i} Z\left(n_{i}\right)^{g}$, for $i=1,2$, even though the government allocates $s_{i} Z$ to group $i$.

The parameter $g$ indicates the magnitude of size effect. In this case, $g$ may represent non-pecuniary psychological prize such as honor or pride. Or $g$ may be viewed as the magnitude of future expected benefit. As the more contenders can signal the potential quality of the winner, this may increase the possibility of sponsorship contracts in the future, for example. The parameter $g$ belongs to the closed interval [0,2] as in Eggert and Kolmar [4] and Lee [3].

In order to analyze the two-stage contest, we solve backwards. That is, utilizing the results derived from the analysis of the second-stage of the contest, we later examine the first-stage inter-group share contest.

\subsection{Second-Stage Contest}

Risk-neutral contender $j$ of group $i$ expends an amount $e_{i j}$ of resource in units commensurate with the prize, for $i=$ 1,2 , and $j=1,2, \cdots, n_{i}$, in the second-stage intra-group contest. Specifically, contender $j$ of group $i$ solves the following problem, for $i=1,2$, and $j=1,2, \cdots, n_{i}$,

$$
\max v_{i j} \equiv p_{i j} s_{i} Z\left(n_{i}\right)^{g}-e_{i j}
$$

where $v_{i j}$ denotes the expected payoff to contender $j$ of group $i$ and $p_{i j}$ is given by $p_{i j}=e_{i j} / \sum_{k} e_{i k}$ Simultaneous solution of the first-order conditions for Equation (1) gives the equilibrium effort $e\left(n_{i}\right)$, the individual payoff

\footnotetext{
${ }^{3}$ The share of group $i$ may also be viewed as the probability that group $i$ wins the entire prize when the prize is indivisible. Under risk neutrality, this assumption can be adopted without affecting the qualitative results of the paper.
}

$v\left(n_{i}\right)$ of each contender, and the group payoffs $w\left(n_{i}\right)$, as follows:

$$
\begin{aligned}
& e\left(n_{i}\right)=\left(n_{i}-1\right)\left(n_{i}\right)^{g-2} s_{i} Z, \\
& v\left(n_{i}\right)=\left(n_{i}\right)^{g-2} s_{i} Z,
\end{aligned}
$$

and

$$
w\left(n_{i}\right)=n_{i} v\left(n_{i}\right)=\left(n_{i}\right)^{g-1} s_{i} Z, \quad \text { for } i=1,2 .
$$

The parameter $g$ does affect the individual payoff as well as the group payoffs. ${ }^{4}$ The payoffs to the contenders increase by the factor of $\left(n_{i}\right)^{g}$ for contenders in group $i$, for $i=1,2$. Note that $e\left(n_{i}\right), v\left(n_{i}\right)$ and $w\left(n_{i}\right)$ all increase in $g$. Moreover, the larger the group, the greater is the size effect, if $g>0$.

\subsection{First-Stage Share Contest}

We now examine the first-stage share contest between the two groups. This paper considers two scenarios of endogenous shares. A natural scenario is that the contenders determine their contribution to the share contest by a non-cooperative Nash strategy, as they do in the second stage. Another scenario is that the contenders in each group cooperate in the share contest. In such a case the representative of each group chooses the level of contribution that maximizes the group payoffs. The shares are determined to be proportional to group contributions in the share contest.

We first consider the case when the contenders employ a non-cooperative Nash strategy. Contender $j$ of group $i$ contributes an amount $x_{i j}$ in units commensurate with the prize to the share contest, for $i=1,2$, and $j=1,2, \cdots, n_{i}$ We denote the group expenditures in the first stage by $X_{1}$ and $X_{2}$. That is, $X_{1}=\sum_{k} x_{l k}$ and $X_{2}=\sum_{k} x_{2 k}$.

Group $i$ 's share is assumed to be given by, for $i=1,2$,

$$
s_{i}(N C)=X_{i} /\left(X_{1}+X_{2}\right),
$$

where $N C$ denotes the solution for the non-cooperative Nash regime. In the first-stage, contender $j$ of group $i$ solves the following maximization problem, for $i=1,2$, and $j=1,2, \cdots, n_{i}$.

$$
\max \left[X_{i} /\left(X_{1}+X_{2}\right)\right] Z /\left(n_{i}\right)^{2}-x_{i j}
$$

Simultaneous solution of the first-order conditions for maximization in the first stage yields the equilibrium group expenditures and the equilibrium shares in the first stage of the non-cooperative Nash regime, for $i, j=1,2$, and $i \neq j$,

$$
X_{i}^{*}(N C)=\left(n_{j}\right)^{2-g} Z /\left[\left(n_{1}\right)^{2-g}+\left(n_{2}\right)^{2-g}\right]^{2}
$$

and

${ }^{4}$ This result is different from the contest with size effect through cost. Interested readers can refer to Lee [3]. 


$$
\begin{aligned}
s_{i}^{*}(N C) & =X_{i}^{*}(N C) /\left[X_{1}^{*}(N C)+X_{2}^{*}(N C)\right] \\
& =\left(n_{j}\right)^{2-g} /\left[\left(n_{1}\right)^{2-g}+\left(n_{2}\right)^{2-g}\right]
\end{aligned}
$$

Equation (5) shows that the smaller group expends more than the larger group in the first stage, and as a result, obtains the larger share when the contenders employ a non-cooperative Nash strategy in the first-stage share contest. However, the size effect through prize mitigates the disadvantage of the larger group, to some extent. The share of the larger group, $s_{1}^{*}(N C)$, increases in $g$. At the maximum of $g=2$, the larger group, group 1, obtains the half of the prize.

Inserting $s_{i}^{*}(N C)$ into Equation (2), we obtain the equilibrium effort of each contender, the individual payoff and the group payoffs:

$$
\begin{aligned}
& e\left(n_{i}, N C\right) \\
& =\left(n_{i}-1\right)\left(n_{i}\right)^{g-2}\left(n_{j}\right)^{2-g} Z /\left[\left(n_{1}\right)^{2-g}+\left(n_{2}\right)^{2-g}\right] \\
& v\left(n_{i}, N C\right)=\left(n_{i}\right)^{g-2}\left(n_{j}\right)^{2-g} Z /\left[\left(n_{1}\right)^{2-g}+\left(n_{2}\right)^{2-g}\right],
\end{aligned}
$$

and

$$
\begin{aligned}
& W\left(n_{i}, N C\right)=\left(n_{i}\right)^{g-1}\left(n_{j}\right)^{2-g} Z /\left[\left(n_{1}\right)^{2-g}+\left(n_{2}\right)^{2-g}\right], \\
& \text { for } i, j=1,2 \text {, and } i \neq j
\end{aligned}
$$

We now examine the inter-group share contest when the contenders in each group respectively cooperate to maximize the group payoffs. In such a case, the representative of group $i$ solves the following maximization problem, for $i=1,2$,

$$
\begin{aligned}
\max W\left(n_{i}\right)-X_{i} & =s_{i} Z / n_{i}-X_{i} \\
& =\left[X_{i} /\left(X_{1}+X_{2}\right)\right] Z / n_{i}-X_{i}
\end{aligned}
$$

Simultaneously solving the first-order conditions for Equation (7), we obtain the equilibrium group expenditures and the equilibrium shares in the cooperative regime, for $i, j=1,2$, and $i \neq j$,

$$
x_{i}^{*}(C)=\left(n_{i}\right)^{1-g} Z /\left[\left(n_{1}\right)^{1-g}+\left(n_{2}\right)^{1-g}\right]^{2},
$$

and

$$
\begin{aligned}
s_{i}^{*}(C) & =X_{i}^{*}(C) /\left[X_{1}^{*}(C)+X_{2}^{*}(C)\right] \\
& =\left(n_{j}\right)^{1-g} /\left[\left(n_{1}\right)^{1-g}+\left(n_{2}\right)^{1-g}\right]
\end{aligned}
$$

It is evident that the size effect increases the share of the larger group. If $g=1$, the two groups obtain the equal share of the prize. Moreover, if $1<g \leq 2$, then the larger group obtains the larger share. Inserting $s_{i}^{*}(C)$ into Equation (2), we obtain the equilibrium effort in the second stage, the individual payoff and the group payoffs in the cooperative regime:

$$
\begin{aligned}
& e\left(n_{i}, C\right) \\
& =\left(n_{i}-1\right)\left(n_{i}\right)^{g-2}\left(n_{j}\right)^{1-g} Z /\left[\left(n_{1}\right)^{1-g}+\left(n_{2}\right)^{1-g}\right] \\
& v\left(n_{i}, C\right)=\left(n_{i}\right)^{g-2}\left(n_{j}\right)^{1-g} Z /\left[\left(n_{1}\right)^{1-g}+\left(n_{2}\right)^{1-g}\right],
\end{aligned}
$$

and

$$
\begin{aligned}
& W\left(n_{i}, C\right)=\left(n_{j}\right)^{1-g} Z /\left[\left(n_{1}\right)^{1-g}+\left(n_{2}\right)^{1-g}\right], \\
& \text { for } i, j=1,2 \text {, and } i \neq j
\end{aligned}
$$

We now compare the non-cooperative Nash regime with the cooperative regime. Under the assumption that $n_{1} \geq n_{2}$ and $N \geq 3$, we obtain:

Remark 1.

1) $s_{1}^{*}(C) \geq s_{1}^{*}(N C)$, where the equality holds when $n_{1}$ $=n_{2}$.

2) $X_{1}^{*}(C)+X_{2}^{*}(C)>X_{1}^{*}(N C)+X_{2}^{*}(N C)$.

3) $n_{1} e\left(n_{1}, C\right)+n_{2} e\left(n_{2}, C\right) \geq n_{1} e\left(n_{1}, N C\right)+n_{2} e\left(n_{2}, N C\right)$, where the equality holds when $n_{1}=n_{2}$.

4) $v\left(n_{1}, C\right) \geq v\left(n_{1}, N C\right)$ and $v\left(n_{2}, C\right) \geq v\left(n_{2}, N C\right)$, where the equalities hold when $n_{1}=n_{2}$.

The size effect through prizes mitigates, to some extent, the group size disadvantage resulting from fierce intra-group competition in the larger group. Moreover, if the size effects are strong enough in the cooperative regime, the larger group can even obtain the larger share. The groups expend more in the first-stage share contest in the cooperative regime than in the non-cooperative Nash regime. This is because the larger group is free of the free-rider problem in the cooperative regime.

The afore-mentioned results draw some important insights regarding government policies. In our set-up, how the contenders in each group perceive the psychological benefit of the size effect has crucial effect on the equilibrium outcomes. If contenders care more about psychological benefit, then the size effects become strong enough and different outcomes may follow. Moreover, different frames may lead to different motives and eventually may induce different equilibrium outcomes. In this case, the role of group leader may be important. For example, if an organization penalizes free riders severely, then the free rider problem is minimized within this organization. The contenders regard as if they are in a cooperative regime rather than in a non-cooperative Nash regime. Therefore, it is important how an organization (or its leader) delivers incentives (psychological benefits, especially) and sets up the frame for its members.

\section{Concluding Remarks}

We have examined prize allocation in contests when the number of contenders affects prizes. We have found the following results. The contenders expend more resources when they cooperate in the inter-group share contest than 
when they contribute non-cooperatively to the intergroup share contest. The size effect through prizes works to mitigate the group size effect, thereby increasing the prize share of the larger group and resulting in more efforts expended in the contest. The larger group can obtain the larger share if the size effect through prizes is strong enough.

This paper can be extended in several aspects. First, the model can provide an important insight on public finance. Public officials have to realize that not only pecuniary but also non-pecuniary prizes are important in the allocation of public funds. If we can explicitly incorporate psychological benefits into the model, then we can draw some interesting policy implications regarding the non-pecuniary prizes. Another theoretical extension is to allow for more than two groups of contenders. In a broader perspective, the present paper is related to the theme of optimal contest design examined by Gradstein and Konrad [6] and Gradstein [7].

\section{Acknowledgements}

We wish to thank Oliver Gürtler and seminar participants at Kookmin University for their valuable comments and suggestions on an earlier version of the manuscript. This research was supported by 2012 Faculty Research Fund of Kookmin University.

\section{REFERENCES}

[1] R. D. Congleton, A. L. Hillman and K. A. Konrad, "40 Years of Research on Rent Seeking 1," Springer-Verlag, Berlin, 2008. doi:10.1007/978-3-540-79247-5

[2] K. A. Konrad, "Strategy and Dynamics in Contests," Oxford University Press, Oxford, 2009.

[3] S. Lee, "Contests with Size Effects through Costs," European Journal of Political Economy, Vol. 23, No. 4, 2007, pp. 1190-93. doi:10.1016/j.ejpoleco.2006.08.002

[4] W. Eggert and M. Kolmar, "Contests with Size Effects," European Journal of Political Economy, Vol. 22, No. 4, 2006, pp. 989-1008. doi:10.1016/j.ejpoleco.2005.06.005

[5] E. Katz and J. Tokatlidu, "Group Competition for Rents," European Journal of Political Economy, Vol. 12, No. 4, 1996, pp. 599-607. doi:10.1016/S0176-2680(96)00027-4

[6] M. Gradstein and K. Konrad, "Orchestrating Rent Seeking Contests," Economic Journal, Vol. 109, 1999, pp. 536-545. doi:10.1111/1468-0297.00461

[7] M. Gradstein, "Optimal Contest Design: Volume and Timing of Rent Seeking in Contest," European Journal of Political Economy, Vol. 14, No. 4, 1998, pp. 575-585. doi:10.1016/S0176-2680(98)00026-3 